\title{
The mechanics of eddy transport from one hemisphere to the other
}

\author{
By NATHAN PALDOR ${ }^{1 *}$, ANDREY SIGALOV ${ }^{1}$ and DORON NOF ${ }^{2}$ \\ ${ }^{1}$ Institute of Earth Sciences, The Hebrew University of Jerusalem, Israel \\ ${ }^{2}$ School of Oceanography, Florida State University, Tallahassee, USA
}

(Received 22 July 2002; revised 6 February 2003)

\section{SUMMARY}

The trajectory of a dense eddy propagating along the bottom of a meridional channel of parabolic crosssection from the southern to the northern hemisphere is described by a Hamiltonian system with two degrees of freedom. Two simplified types of motion exist in which the meridional acceleration vanishes: in midlatitudes the motion is geostrophic, poleward (equatorward) directed along the western (eastern) flank of the channel, while on the equator the motion consists of zonal oscillations along the potential-well generated by the bottom parabolic cross-section of the channel. The eddy's propagation along the equator is much faster than that in midlatitudes, which enhances its dissipation via mixing with the overlying ocean water. For motions that occur slightly off the equator the eastward segment is stable while the westward segment is unstable, so an expulsion from the equatorial regime takes place during the latter. A dense eddy that arrives near the equator along the west flank of the channel, has to cross the channel to its east flank where it can either oscillate back (westward) to the other side, or move poleward from the equator along the channel's east flank. The eddy's dissipation during the equatorial part of its trajectory is very large, and the probability of the dissipated eddy leaving the equator to either hemisphere is identical.

The non-integrability of the system is manifested in the sensitive combination of the equatorial and the midlatitude regimes that renders the dynamics of the transport of dense eddies across the equator chaotic. This description explains both the sharp decrease in the amount of Antarctic bottom water mass in the immediate vicinity of the equator in the western Atlantic Ocean and the 'splitter' effect of the equator. This effect, encountered in earlier fluid dynamical numerical simulations, causes a current, and a cloud of particles, to chaotically split into two parts flowing in different hemispheres.

KEYWORDs: AABW transport Cross-equatorial flow Non-integrable Hamiltonian dynamics

\section{INTRODUCTION}

The precise way in which the dense Antarctic Bottom Water (AABW) makes its way from Antarctica, across the equator and into the northern hemisphere along the ocean floor has intrigued oceanographers for over four decades. Conjecture regarding the existence of deep (below $2000 \mathrm{~m}$ ) equatorward flow along the ocean floor was first raised by Stommel (1958), based only on a simple thermally driven convective model and general consideration regarding the distribution of oxygen and temperature in the deep ocean. The dynamics of this slow, but high-transport, flow (about $0.03 \mathrm{~cm} \mathrm{~s}^{-1}$ and $50 \mathrm{~Sv}$ according to Stommel) has been the subject of numerous theoretical, computational and observational works that have all tried to better quantify and ascertain its existence. Even though there have not been any direct observations of eddies on the ocean bottom, it makes sense to assume that some of the transport is discontinuous and that, just like the upper ocean, some of the transport probably occurs via eddies (see e.g. Borisov and Nof 1998). Our goal in this work is to analyse the dynamics of the transport of dense eddies from high latitudes of the southern hemisphere into the northern hemisphere from a mechanical viewpoint, and to explain several features of this transport that are directly observed or numerically computed.

Observational and theoretical advances made during the last four decades are summarized in Nof and Borisov (1998, hereafter NB) to which we refer the reader for a more complete review of the subject. The present study complements the theoretical works of NB and Borisov and Nof (1998) by casting both the equatorward motion from

\footnotetext{
* Corresponding author: Institute of Earth Sciences, The Hebrew University of Jerusalem, Jerusalem, 91904 Israel. e-mail: nathan.paldor@huji.ac.il
}

(c) Royal Meteorological Society, 2003. 
high southern latitudes and the equator-crossing problems into Hamiltonian systems. As was shown in NB, particle Lagrangian formulation of the equator-crossing problem by deep ocean eddies yields similar results to those obtained from simulations of a continuous current by an Ocean General Circulation Model (OGCM). This result lends credence to the notion, adopted in the present study, that a particle analysis is relevant to the transport of dense water to the northern hemisphere. In addition, the motion of eddies is best described by following their centre-of-mass, so if dense water is transported by eddies, a particle model (where the azimuthal velocity de-couples from the translation) is the most appropriate approach.

We now briefly review the elements of a dense-water transport scenario relevant to analytical mechanics.

\section{(a) Equatorward flow from Antarctica}

A precise, widely accepted definition of AABW characteristics does not exist in the literature, but the term is broadly attributed to potential temperature of less than $1.9^{\circ} \mathrm{C}$ at depths exceeding $4000 \mathrm{~m}$ (Whitehead and Worthington 1982). As is clearly evident from the meridional sections shown in their work (see their Fig. 1), AABW occupies the depths exceeding $4000 \mathrm{~m}$ in the western North Atlantic as far north as latitude $40^{\circ} \mathrm{N}$. (Strictly speaking this water is actually 'modified Antarctic water' as the water has been modified by mixing during its long northward flow from the South Atlantic, but the term $\mathrm{AABW}$ has been used for very long time and, besides, the Antarctic origin of the cold water justifies its continued usage.) The potential temperature of the overlying North Atlantic Deep Water is more than $2{ }^{\circ} \mathrm{C}$, and it occupies depths between 1500 and $4000 \mathrm{~m}$ in the western North Atlantic so vertical mixing entails a downward flux of heat.

The inertial current model of Stommel and Arons (1960a,b) views the AABW flow as a western boundary current flowing equatorward along the flank of a channel that extends from high latitudes of the southern hemisphere to the equator. This theory deals only with the flow of (a column of) water from its source near the Pole to the vicinity of the equator, and ignores the equator crossing into the other hemisphere.

A straightforward consideration of angular momentum conservation indicates that a parcel of AABW originating at, say, latitude $55^{\circ} \mathrm{S}$ with zero initial zonal velocity has increased its angular momentum, upon reaching the equator, by an amount equivalent to an increase of its zonal velocity to $200 \mathrm{~m} \mathrm{~s}^{-1}$. How does this change of angular momentum take place without a corresponding increase in its kinetic energy? A careful account of the changes in angular momentum that a deep-water eddy undergoes on its way from Antarctica to the equator is the first issue addressed by the present study.

\section{(b) Equator crossing}

The process of equator-crossing by an AABW water parcel is not addressed by the Stommel and Arons theory, but subsequent theoretical work on the subject stresses the possible role of either dissipation or relative vorticity changes along the western boundary. The reader is referred to NB for a thorough review of this important issue. Despite the basic differences between the dynamics of isolated eddies, modelled as point masses, and that of continuous currents, many of the details related to their equatorcrossing were shown by NB to be similar. Specifically, the simulation both of particles and continuous currents shows that the equator acts as a 'splitter', in the sense that in both cases a fraction of the particles/current does not cross the equator into the other hemisphere and, instead, recirculates in its hemisphere of origin. A careful analysis of the dynamics associated with this 'splitting' of meridionally moving particles/current at 
the equator is the second focus of our study, both of which are addressed in the context of particle dynamics.

The common thread that connects the two foci of the present paper is the transport of AABW into the northern hemisphere. The meridional cross-section of Whitehead and Worthington (1982) indicates that the AABW core (i.e. water with potential temperature less than about $1^{\circ} \mathrm{C}$ ) is entirely missing north of the equator. The NB compilation of data quantifies this diminishing of the transport with latitude in the Atlantic Ocean, and shows that the decrease of this transport between $30^{\circ} \mathrm{S}$ and the equator is not uniform. From $7 \mathrm{~Sv}$ at the south end of the Brazil basin $\left(\sim 30^{\circ} \mathrm{S}\right)$ the AABW transport decreases only slightly to $6.7 \mathrm{~Sv}$ at $23^{\circ} \mathrm{S}$ and to $5.5 \mathrm{~Sv}$ at $11^{\circ} \mathrm{S}$ - for a total decrease of $20 \%(1.5 \mathrm{~Sv})$ in over $20^{\circ}$ of latitude. In contrast, upon reaching the vicinity of the equator the AABW transport decreases abruptly to about $2 \mathrm{~Sv}$ only-a decrease of $65 \%(3.5 \mathrm{~Sv})$ in just a few degrees of latitude! The data of Whitehead and Worthington (1982) show that the rapid decrease in the AABW transport continues into the northern hemisphere and at $2^{\circ} \mathrm{N}$ it is only a fraction of $1 \mathrm{~Sv}$. The scenarios in the Pacific and Indian Oceans are not as well documented as in the Atlantic Ocean, but the available scant observations indicate a qualitatively similar picture. This observation supports the theoretical conclusion reached by NB, that the equator acts as a 'splitter' of the AABW current as it does for a cloud of particles. The small decrease of AABW transport in the $20^{\circ}$ of latitudes south of $11^{\circ} \mathrm{S}$ can be attributed to mixing with the overlying water, but the larger decrease near the equator is, presumably, caused by the additional 'equatorial effect'.

The two issues addressed in the present work - the changes in angular momentum that occur when water columns flow from Antarctica to the equator and the dynamics of equator crossing along the ocean's bottom — are both studied by substituting the angular momentum for the zonal velocity component in the governing Lagrangian equations. This, in turn, allows the construction of a canonical Hamiltonian form for the two degrees of freedom (2DOF) dynamical system. The same substitution was employed in the study of cross-equatorial flow under prescribed meridional potential (Dvorkin and Paldor 1999), and for quantifying the zonal drift of time-dependent free particle motion on the surface of the rotating earth (Paldor 2001).

This paper is organized as follows: in section 2 we develop the Hamiltonian form of the system that describes the motion of a deep-water eddy along the bottom of a parabolic meridional channel. This form of the dynamics is applied in section 3 to the equatorward motion from Antarctica to the equator, by combining analytical results with numerical integration of the equations in midlatitudes. The same form is applied in section 4 to the equator-crossing process, as well as to the details of the splitter role of the equator. The two regimes are combined in section 5 to the entire trajectory of an AABW eddy from Antarctica to the equator and continuing from there to either of the two hemispheres.

\section{HAMILTONIAN FORM OF THE TRANSPORT EQUATIONS AND ITS ANALYSIS}

Consider the geometry shown in Fig. 1 where a deep (i.e. cold water) eddy moves along the bottom of a channel whose axis is directed in the meridional direction $(\phi)$ and whose depth is parabolic in the longitudinal direction $(\lambda)$. We regard the eddy's centre of mass as a particle that obeys the momentum equations:

$$
\begin{gathered}
U_{t}=V \sin (\phi)(2 \Omega+U / R \cos (\phi))-g^{\prime} h_{\lambda} / R \cos (\phi), \\
V_{t}=-U \sin (\phi)(2 \Omega+U / R \cos (\phi)),
\end{gathered}
$$




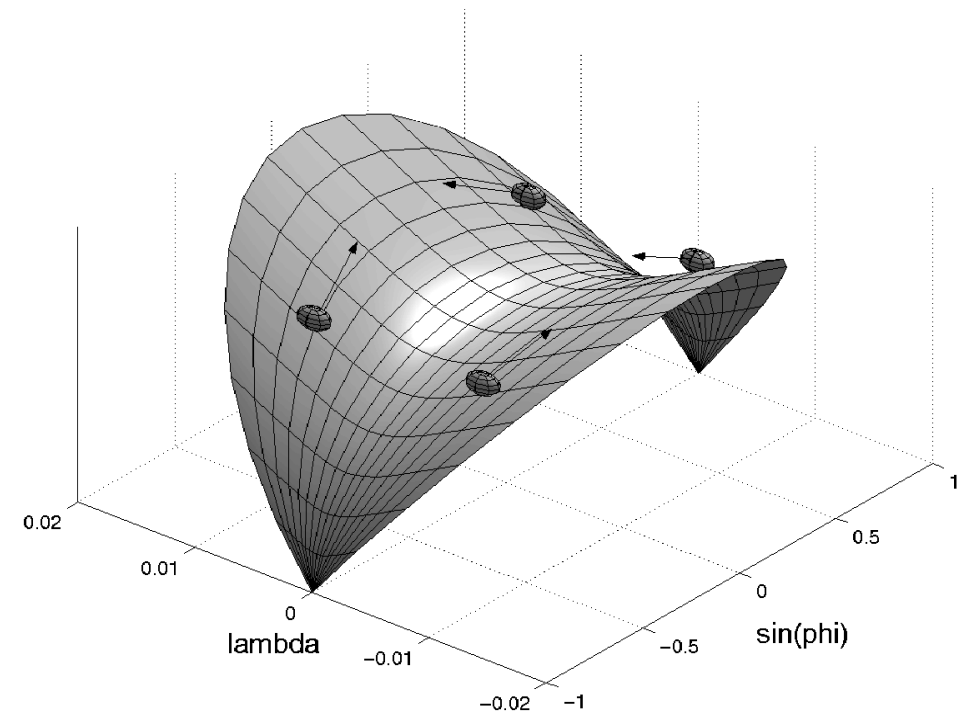

Figure 1. The geometry of the meridional, parabolic channel on the spherical earth, showing latitude $(\lambda)$ and sine longitude $(\phi)$. The earth itself is missing from the figure and only the channel (with schematic deep water eddies) is shown. The longitude scale is arbitrary (bottom shoaling depends on $\Delta \lambda$ ) and the vertical axis is the height from the earth's centre, i.e. the ratio of channel depth to earth's radius is greatly exaggerated.

where $U$ and $V$ are the velocity components of the eddy's centre of mass in the zonal $(\lambda)$ and meridional $(\phi)$ directions, respectively, $\Omega$ and $R$ are the earth's rotation rate and radius, respectively, $g^{\prime}$ is the reduced gravity $\left(g^{\prime}=g\left(\rho_{1}-\rho_{2}\right) / \rho_{1}\right.$, with $g$ the gravitation constant, $\rho_{1}$ and $\rho_{2}$ are the densities of the water in the eddy and in the overlying water, respectively) and $h(\lambda)$ is the relief of the channel bottom measured relative to the centreline, $\lambda=0$. The bottom relief is assumed to be parabolic, $h(\lambda)=H(\lambda / \Delta \lambda)^{2}$, so $h(\lambda)$ increases from $h(0)=0$ to $h=H$ at $|\lambda|=\Delta \lambda$. Subscripts of variables indicate derivatives (e.g. $\lambda$ in (2.1a)) and time-derivatives on the left-hand side (1.h.s.) are Lagrangian derivatives. In these equations both the variation of the Coriolis parameter, $2 \Omega \sin (\phi)$, with $\phi$ and the curvature of the earth's surface (via the metric terms $U /(R \cos (\phi)))$ are fully taken into account. In the following, the subscript 's' will indicate a special (e.g. steady) solution. The momentum equations $(2.1 \mathrm{a}, \mathrm{b})$ extend the momentum equations on the equatorial $\beta$-plane, studied in NB, to the global scale.

In addition to these momentum equations, the eddy's coordinates vary with time as:

$$
\begin{gathered}
\lambda_{t}=U / R \cos (\phi), \\
\phi_{t}=V / R .
\end{gathered}
$$

As in Dvorkin and Paldor (1999), see also Paldor and Killworth 1988), the set (2.1) is non-dimensionalized so as to minimize the number of system parameters. The lengthand time-scales that appear in system $(2.1)-R$ and $(2 \Omega)^{-1}$-provide the scales for non-dimensionalizing the corresponding variables and the resulting velocity scale is $(2 \Omega R) \approx 930 \mathrm{~m} \mathrm{~s}^{-1}$. Thus, a dimensional velocity of $10 \mathrm{~cm} \mathrm{~s}^{-1}$ corresponds to a nondimensional velocity of about $10^{-4}$. 
The non-dimensional form of system (2.1) is:

$$
\begin{gathered}
U_{t}=V \sin (\phi)(1+U / \cos (\phi))-\alpha \lambda / \cos (\phi), \\
V_{t}=-U \sin (\phi)(1+U / \cos (\phi)), \\
\lambda_{t}=U / \cos (\phi), \\
\phi_{t}=V
\end{gathered}
$$

where $U, V$ and $t$ are now dimensionless and $\alpha=2 g^{\prime} H /(2 \Omega R \Delta \lambda)^{2}$. For typical oceanic values of $g^{\prime}=0.5 \times 10^{-2} \mathrm{~m} \mathrm{~s}^{-2}, H=2000 \mathrm{~m}$ and $\Delta \lambda=0.5$ radian, $\alpha$ is of order $10^{-4}$. Note that $\alpha$ is the single parameter of system (2.2) that augments the five dimensional parameters $\left(g^{\prime}, R, \Omega, H, \Delta \lambda\right)$ of system $(2.1)$.

System (2.2) conserves energy but does not have a canonical Hamiltonian form, which is obtained next.

\section{(a) Canonical form of the momentum equations}

To write system (2.2) in canonical form we substitute the angular momentum for the zonal velocity. In non-dimensional form, the angular momentum, $D$, is:

$$
D=\cos (\phi)(\cos (\phi) / 2+U),
$$

so that the zonal velocity can be recovered from the value of $D$ via:

$$
U=D / \cos (\phi)-\cos (\phi) / 2 \text {. }
$$

Expressing the total energy in terms of $D$ instead of $U$ the Hamiltonian function is:

$$
\mathrm{H}(V, \phi, D, \lambda)=\frac{1}{2} V^{2}+\frac{1}{2}(D / \cos (\phi)-\cos (\phi) / 2)^{2}+\frac{1}{2} \alpha \lambda^{2},
$$

and $(V, \phi)$ and $(D, \lambda)$ are its two pairs of conjugate variables satisfying the canonical equations:

$$
\begin{gathered}
\phi_{t}=V=\mathrm{H}_{V}, \quad V_{t}=\frac{1}{2} \sin (2 \phi)\left(\frac{1}{4}-D^{2} / \cos ^{4}(\phi)\right)=-\mathrm{H}_{\phi} ; \\
\lambda_{t}=D / \cos ^{2}(\phi)-\frac{1}{2}=\mathrm{H}_{D}, \quad D_{t}=-\alpha \lambda=-\mathrm{H}_{\lambda} .
\end{gathered}
$$

Set (2.6) is the sought canonical form of the eddy translation model and the Hamiltonian (2.5) is its integral of motion. Thus, when $\mathrm{H}$ is the only integral, the system is not integrable so chaotic bands exist. In contrast, when the channel's bottom is flat $(h(\lambda)=$ constant) or when gravity is set equal to zero $(\alpha=0), D$ is also conserved according to (2.6b), so the $2 \mathrm{DOF}$ system (2.6) has two integrals of motion, $\mathrm{H}$ and $D$, i.e. the system is integrable. This case is a natural starting point for the analyses of (2.6) since many of its dynamical features already exist in the inertial system, which is briefly summarized next.

\section{(b) The inertial dynamics}

Setting $\alpha=0$ in system (2.6) results in the inertial system, studied thoroughly in Paldor (2001) and Paldor and Sigalov (2001). Equation (2.6b) implies that $D$ is a parameter of the integrable $1 \mathrm{DOF}$ system (2.6a). The cyclic coordinate $\lambda(t)$ is determined by $D$ and $\phi(t)$ via $\lambda_{t}=D / \cos ^{2}(\phi(t))-\frac{1}{2}$, and The Hamiltonian (2.5) is:

$$
\mathrm{H}_{\text {inertial }}(V, \phi ; D)=\frac{1}{2}\left\{V^{2}+(D / \cos (\phi)-\cos (\phi) / 2)^{2}\right\} .
$$

(Note: $D$ is a variable in 2.5 but it appears as a parameter in 2.7 though $\lambda_{t}=$ $\partial \mathrm{H}_{\text {inertial }} / \partial D$ !) 
For $D>\frac{1}{2}$ the origin, $V=0=\phi$, is the only fixed point of the reduced $(V, \phi)$ system and the associated zonal motion along the equator is directed eastward with constant speed $U=D-\frac{1}{2}>0$. Near this fixed point the $(V, \phi)$ motion consists of oscillations with frequency $\left(D^{2}-\frac{1}{4}\right)^{\frac{1}{2}}$. The averaged (with respect to the $\lambda(t)$ oscillation that follows from the $(V, \phi)$ oscillation) rate of longitude increase, $\langle\mathrm{d} \lambda / \mathrm{d} t\rangle$, is given by:

$$
\langle\mathrm{d} \lambda / \mathrm{d} t\rangle=\left(D-\frac{1}{2}\right)+D \cdot \delta E /\left(D^{2}-\frac{1}{4}\right),
$$

where $\delta E=\left.\frac{1}{2} V^{2}\right|_{\phi=0}$ is the difference between the value of $\mathrm{H}_{\text {inertial }}(V, \phi ; D)$ and $\frac{1}{2}\left(D-\frac{1}{2}\right)^{2}=\mathrm{H}_{\text {inertial }}(0,0 ; D)$. This averaged longitude increase has two contributions: the first, $D-\frac{1}{2}=U>0$, is due to the eastward zonal velocity along the equator (see (2.4)); the second term is due to the drift associated with the averaged oscillatory motion (over the period $\left.T=2 \pi /\left(D^{2}-\frac{1}{4}\right)^{\frac{1}{2}}\right)$, which is also directed eastward for $D>\frac{1}{2}$.

For $D<\frac{1}{2}$ (i.e. $\left.U\right|_{\phi=0}<0$ ) the equator is a hyperbolic fixed point of (2.6a), so the linearized motion near it is exponential in time (i.e. westward motion on the equator is unstable) and two elliptic fixed points occur at latitudes $\phi_{\mathrm{ell}}= \pm \operatorname{arcos}\left\{(2 D)^{\frac{1}{2}}\right\}$. The analysis near these points $\left(V_{\text {ell }}=0, \phi_{\text {ell }}= \pm \operatorname{arcos}\left\{(2 D)^{\frac{1}{2}}\right\}\right)$ shows that the frequency of $(V, \phi)$ oscillation near them equals $\sin \left(\phi_{\mathrm{ell}}\right)$ (the non-dimensional Coriolis frequency), so the period of these inertial, midlatitude, oscillations is:

$$
T=2 \pi / \sin \left(\phi_{\mathrm{ell}}\right)=2 \pi(1-2 D)^{-\frac{1}{2}} .
$$

The zonal motion $\lambda(t)$ near these midlatitude fixed points consists of oscillations with period given by $(2.9)$ due to the oscillations in $(V, \phi)$ and a westward-directed drift at a rate given by:

$$
\langle\mathrm{d} \lambda / \mathrm{d} t\rangle=-\delta E / \sin ^{2}\left(\phi_{\mathrm{ell}}\right)=-\delta E(1-2 D)^{-1} .
$$

Here, $\delta E$ is the value of $\mathrm{H}_{\text {inertial }}(V, \phi ; D)$ (recall that $\mathrm{H}_{\text {inertial }}\left(0, \phi_{\text {ell }} ; D\right)=0.0$ ) and, since the Hamiltonian is conserved, $\delta E$ is determined by the initial conditions of $V(0)$, $\phi(0)$ and $U(0)$ (i.e. by $V(0), \phi(0)$ and $D)$. To sum up the relevant aspects of inertial dynamics: there exist two oscillatory regimes, an equatorial regime where the eddy oscillates between the two sides of the equator while drifting eastward, and a midlatitude regime where the eddy oscillates about a mean latitude in one hemisphere, while drifting westward.

\section{(c) Special solutions}

The special solutions of system (2.6) considered here (designated by the subscript 's') obtain by requiring that $V_{t}$ vanishes identically. Steady solutions of (2.6) are a particular type of special solutions obtained by setting $\partial / \partial t=0$ in all four equations, and a straightforward calculation shows that (for realistic $U$ and $\phi$, i.e. non-negative $D$ ) these solutions satisfy:

$$
V_{\mathrm{s}}=0=\lambda_{\mathrm{s}} ; \quad D_{\mathrm{s}}\left(\phi_{\mathrm{s}}\right)=\frac{1}{2} \cos ^{2}\left(\phi_{\mathrm{s}}\right) ; \quad \text { for any (constant) } \phi_{\mathrm{s}} .
$$

In these steady solutions the eddy rests at some latitude $\phi_{\mathrm{s}}$ on the channel's centre-line with zero kinetic energy. Since both $U_{\mathrm{s}}=0$ (see Eq. (2.4)) and $V_{\mathrm{s}}=0$ the eddy remains in this location at all times. Since $D_{\mathrm{s}}=\frac{1}{2} \cos ^{2}\left(\phi_{\mathrm{s}}\right)<\frac{1}{2}$, the analysis of Dvorkin and Paldor (1999) guarantees that all steady states are elliptic in $(V, \phi)$ phase space, so $V$ 
and $\phi$ can only oscillate with frequency $\sin \left(\phi_{\mathrm{s}}\right)$ near $\left(V_{\mathrm{s}}, \phi_{\mathrm{s}}\right)$. Equation (2.6b) ensures that for fixed $\phi=\phi_{\mathrm{s}}$ the linearized dynamics in $(D, \lambda)$ is oscillatory too (with frequency $\left.\alpha^{\frac{1}{2}} / \cos \left(\phi_{\mathrm{s}}\right)\right)$.

The first, non-steady, special solution of (2.6) represents equatorial oscillations. Thus, setting $\phi(t)=0$ in the $V_{t}$ equation of (2.6a) to ensure that the eddy remains on the equator, and $V(t)=0$, at all times one gets:

$$
\begin{gathered}
V_{\mathrm{s}}(t)=0 ; \quad \phi_{\mathrm{s}}(t)=0 ; \quad \partial D_{\mathrm{s}}(t) / \partial t=\partial U_{\mathrm{s}}(t) / \partial t=-\alpha \lambda_{\mathrm{s}}(t) ; \\
\partial \lambda_{\mathrm{s}}(t) / \partial t=\left(D_{\mathrm{s}}(t)-\frac{1}{2}\right)=U_{\mathrm{s}}(t) .
\end{gathered}
$$

This special solution describes harmonic oscillations of $\left(D_{\mathrm{s}}, \lambda_{\mathrm{s}}\right)$ on the equator (where the Coriolis force vanishes so no $V_{\mathrm{s}}$ is generated by the finite $\left.U_{\mathrm{s}}(t)=D_{\mathrm{s}}(t)-\frac{1}{2}\right)$. The Hamiltonian of this system is a 'potential well', $\mathrm{H}\left(U_{\mathrm{s}}, \lambda_{\mathrm{s}}\right)=\frac{1}{2}\left(U_{\mathrm{s}}^{2}+\alpha \lambda_{\mathrm{s}}^{2}\right)=$ $\frac{1}{2}\left\{\left(D_{\mathrm{s}}-\frac{1}{2}\right)^{2}+\alpha \lambda_{\mathrm{s}}^{2}\right\}$, and the frequency of these equatorial oscillations is $\alpha^{\frac{1}{2}}$. The conservation of energy implies that the zonal speed, $\left|U_{\mathrm{S}}(t)\right|$, is determined by $\left|\lambda_{\mathrm{s}}(t)\right|$ via $\left|U_{\mathrm{s}}(t)\right|=\left(2 E-\alpha \lambda_{\mathrm{s}}(t)^{2}\right)^{\frac{1}{2}}$, where $E$ is the value of $\mathrm{H}$ (i.e. either $\left.\frac{1}{2} U_{\mathrm{s}}^{2}\right|_{\lambda=0}$ or $\left.\frac{1}{2} \alpha \max \left\{\lambda_{\mathrm{s}}(t)^{2}\right\}\right)$, i.e. $-(2 E)^{\frac{1}{2}} \leqslant U_{\mathrm{s}}(t) \leqslant(2 E)^{\frac{1}{2}}$.

For $0<\left|V_{\mathrm{s}}\right| \ll\left|U_{\mathrm{s}}\right|$ in system (2.6) $\phi(t)$ does not vanish identically but one can naively deduce that the special solution, (2.12), describes the dynamics to first order in $\phi$ only. However, we will shortly show that this solution is unstable so small deviations from the special solution are repelled from the equator.

The second, non-steady, special solution of (2.6) approximates the midlatitude geostrophic motion. It obtains by setting $D_{\mathrm{s}}=\frac{1}{2} \cos ^{2}\left(\phi_{\mathrm{s}}\right)$ to ensure $V_{t}=0$ in (2.6a) so $V_{\mathrm{s}}(t)$ is constant, not necessarily zero. For oceanic speeds of order $10 \mathrm{~cm} \mathrm{~s}-1$ $\left|V_{\mathrm{s}}\right|=\mathrm{O}\left(10^{-4}\right) \ll 1$ so that terms proportional to $V_{\mathrm{s}}^{2}$ are second order only. Since $D_{\mathrm{s}}=\frac{1}{2} \cos ^{2}\left(\phi_{\mathrm{s}}\right)$ the value of $\lambda_{\mathrm{s}}$ obtains from the $D_{t}$ equation in (2.6b):

$$
\lambda_{\mathrm{s}}=-D_{t} / \alpha=\left(\frac{1}{2 \alpha}\right) \sin \left(2 \phi_{\mathrm{s}}\right) V_{\mathrm{s}} .
$$

Note that differentiation of (2.13) with respect to time yields $\partial \lambda_{\mathrm{s}} / \partial t=\cos \left(2 \phi_{\mathrm{s}}\right) V_{\mathrm{s}}^{2} / \alpha$, which is quadratic in $V_{\mathrm{s}}$ : positive (eastward) for $\phi_{\mathrm{s}}<45^{\circ}$ and negative (westward) for $\phi_{\mathrm{s}}>45^{\circ}$. It follows from (2.13) that $\sin \left(\phi_{\mathrm{s}}\right) V_{\mathrm{s}}=\alpha \lambda_{\mathrm{s}} / \cos \left(\phi_{\mathrm{s}}\right)$, which is simply the geostrophic balance between the zonal pressure gradient at $\lambda_{\mathrm{s}}$ (see (2.2a)) and the Coriolis force, $\sin \left(\phi_{\mathrm{s}}\right) V_{\mathrm{s}}$.

To sum up, to first order in $\left|V_{\mathrm{s}}\right|$, the special midlatitude solution is:

$$
\begin{aligned}
V_{\mathrm{s}} & =2 \alpha \lambda_{\mathrm{s}} / \sin \left(2 \phi_{\mathrm{s}}\right)=\mathrm{constant} ; \quad \phi_{\mathrm{s}}(t)=\phi(0)+V_{\mathrm{s}} t ; \\
D_{\mathrm{s}}\left(\phi_{\mathrm{s}}\right) & =\frac{1}{2} \cos ^{2}\left(\phi_{\mathrm{s}}\right) ; \lambda_{\mathrm{s}}=\sin \left(2 \phi_{\mathrm{s}}\right) V_{\mathrm{s}} /(2 \alpha),
\end{aligned}
$$

so $V_{\mathrm{S}}$ is geostrophic (constant!) and $\phi_{\mathrm{S}}(t)$ and $D_{\mathrm{S}}(t)$ are linear-while $\lambda_{\mathrm{S}}(t)$ is quadratic-in $V_{\mathrm{s}}$.

This special solution is realized only for $V(0)=V_{\mathrm{s}}$. It will be approximately realized for $V(0)=0$ provided $V_{\mathrm{s}} \ll 1$ (see Fig. 2). However, if $V_{\mathrm{s}}$ is not small or $V(0)=0$ this solution does not provide a reasonable approximation and an averaged trajectory is now derived for this case.

\section{(d) Averaged (slow) trajectory}

In addition to the two special solutions found above, an averaged trajectory can be constructed analytically in which the fast oscillatory part of the trajectory (that originates 
from the inertial motion) is filtered out and the slow monotonic motion is the sole contributor to the trajectory. Paldor (2001) applied the same averaging out of the fast oscillations from the zonal drift associated with the inertial oscillations.

In the present problem the averaged trajectory obtains by realizing that for fixed $D<\frac{1}{2}$ the (inertial) oscillations in $(V, \phi)$ have, according to (2.9), a frequency of $(1-2 D)^{\frac{1}{2}}=\sin \left(\phi_{\mathrm{ell}}\right)$. For fixed $\phi$, on the other hand, the frequency of oscillation in $(D$, $\lambda$ ), is $\alpha^{\frac{1}{2}} / \cos (\phi)$ (see 2.6b). Since in midlatitudes, $\sin \left(\phi_{\text {ell }}\right) \sim 0.5$, while $\alpha^{\frac{1}{2}} / \cos (\phi) \sim$ $10^{-2}$ for realistic values in the ocean, we can safely assume that $D$ is constant in the course of a single $(V, \phi)$ oscillation. Thus, when $D$ is fixed the formula for the zonal drift (that filters out the $(V, \phi)$ oscillations from the inertial trajectory) developed in Paldor (2001; his Eq. 5.8) applies straightforwardly in the present case. Accordingly, the coordinates $D^{\text {av }}(t)$ and $\lambda^{\text {av }}(t)$ of the averaged trajectory satisfy:

$$
\lambda^{\mathrm{av}}(t)_{t}=-E /\left(1-2 D^{\mathrm{av}}\right) ; \quad D^{\mathrm{av}}(t)_{t}=-\alpha \lambda^{\mathrm{av}},
$$

where $E=\mathrm{H}-\frac{1}{2} \alpha\left(\lambda^{\mathrm{av}}\right)^{2}$. The latitude's temporal changes are determined from $\cos ^{2}$ $\left(\phi^{\mathrm{av}}(t)\right)=2 D^{\mathrm{av}}(t)$ and the calculation of $V(t)$ is redundant. This averaged trajectory extends the second special solution to the case when the initial velocity $V(0)$ differs significantly from $V_{\mathrm{s}}=2 \alpha \lambda(0) / \sin (2 \phi(0))$.

\section{(e) Stability of special solutions}

An important characteristic of any solution of a dynamical system is its stability, which determines the temporal evolution of small amplitude deviations (perturbations) from it. In order to assess the stability of a special solution, one has to linearize system (2.6) near it and assume that the temporal evolution of the perturbations (to all four variables) is given by $\mathrm{e}^{\mu t}$ where $\mu$ can be complex. When $\operatorname{Re}\{\mu\}$ is positive, the perturbations grow exponentially in time, so after some finite time the perturbations are no longer small.

Applying this procedure to system (2.6) one gets a quadratic equation for $\mu^{2}$, which has to be negative for stability. The condition $\mu^{2}<0$ is guaranteed provided:

$$
4\left(D_{\mathrm{s}}\right)^{2} \tan \left(\phi_{\mathrm{s}}\right)^{4}>\cos \left(2 \phi_{\mathrm{s}}\right)\left(\frac{1}{4}-\left(D_{\mathrm{s}}\right)^{2} / \cos \left(\phi_{\mathrm{s}}\right)^{4}\right) .
$$

This condition is always satisfied by the steady states for $\phi_{\mathrm{s}} \neq 0$, by the midlatitude (i.e. the second non-steady) special solution and by the average trajectory, since in all these cases $\left(\frac{1}{4}-D_{\mathrm{s}}^{2} / \cos \left(\phi_{\mathrm{s}}\right)^{4}\right)=0$ while the 1.h.s. of Eq. (2.16) is positive. However, in the equatorial special solution the 1.h.s. vanishes $\left(\phi_{\mathrm{s}}=0\right)$, while if $D_{\mathrm{s}}<\frac{1}{2}$ the r.h.s. is positive so condition (2.16) is violated. Thus, westward equatorial motion ( $\left.U=D-\frac{1}{2}<0\right)$ is unstable, so the trajectory is repelled from the equator; while for eastward motion ( $U=D-\frac{1}{2}>0$ ) the r.h.s. of Eq. (2.16) is negative, so the solution is stable and the eddy remains close to the equator. These results are invoked in the following two sections to explain both focal issues of this study.

\section{Midlatitude Motion}

The first issue addressed in this work is the dynamical description of the trajectory of a dense eddy from Antarctica to the tropics and the angular momentum balance along this trajectory.

The special solution given by Eq. (2.14) applies to midlatitude, geostrophic, frictionless motions. To first order, the velocity in this special solution is geostrophic 


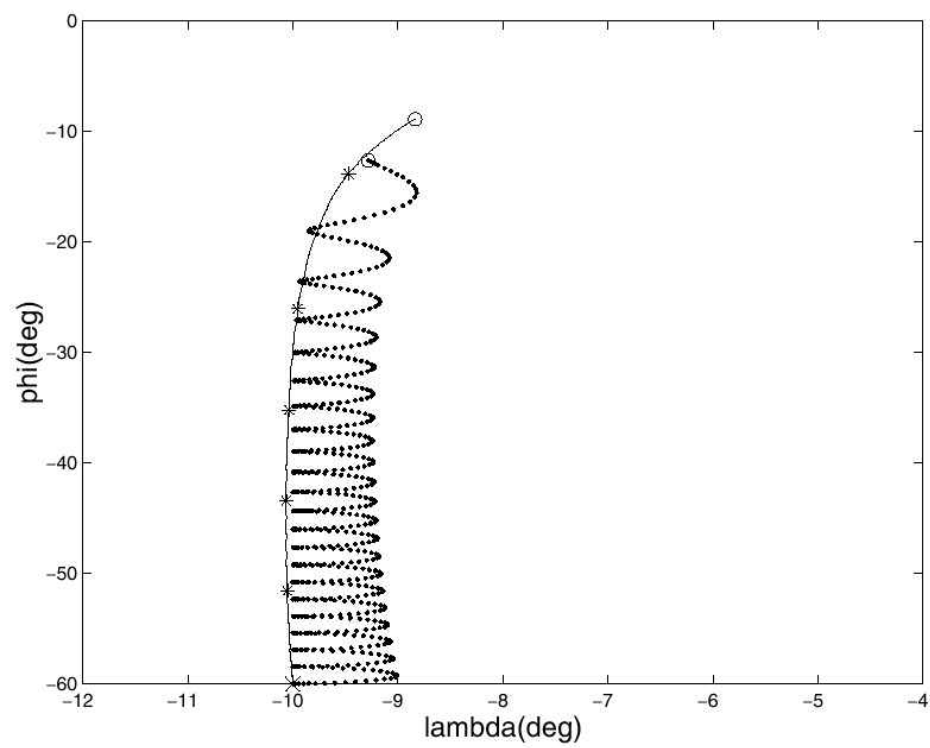

Figure 2. The solution of Eq. (2.6) (dotted curve) and its geostrophic midlatitude approximation Eq. (2.14) (solid curve) starting from the initial conditions: latitude $\phi(0)=60^{\circ} \mathrm{S}$; longitude $\lambda(0)=-10^{\circ}$; velocity components $U(0)=0$ and $V(0)=0$ for the exact solution (dotted curve), and $V(0)=2 \alpha \lambda(0) / \sin (\phi(0))$ for the geostrophic solution. Note that a solution originating near the geostrophic special solution remains close to it at all times. Final time (marked by small open circles) is 210 (2 weeks), stars mark 40 time-unit (3-day) intervals, an 'x' marks the point at $t=0$ and $\alpha=0.01$ (thus the high speed). Similar results obtain for other $\alpha$-values or initial conditions. The second order westward (eastward) drift poleward (equatorward) of the $45^{\circ}$ latitude is clearly evident in the two curves.

and meridionally directed, $V=2 \alpha \lambda / \sin (2 \phi)$; the Coriolis force, $V \sin (\phi)$, balances $\alpha \lambda / \cos (\phi)$, the gravitational force due to the sloping channel. An angular momentum view of this motion is that $D$ increases continuously by the applied zonal pressure gradient force, $D_{t}=-\alpha \lambda$, but since the total energy is not altered, this change in $D$ is manifested only in the continuous change in $\cos (\phi)$ and not in $U$. In addition to the geostrophic meridional velocity, there exists a second order contribution to the cross-channel, zonal, motion given by $\lambda_{t}=\cos (2 \phi) V^{2} / \alpha$, directed westward at latitudes higher than $45^{\circ}$ and eastward at latitudes lower than $45^{\circ}$.

These results are now confirmed by comparing them to numerically computed solutions of (2.2).

\section{(a) Numerical confirmation}

System (2.2) was integrated numerically using a fifth order Runge-Kutta scheme with a $10^{-9}$ tolerance to verify the analytical considerations and to compute the geographical, $(\lambda, \phi)$, trajectories' characteristics. Figure 2 shows the trajectories obtained from the initial conditions: $U(0)=0$ (i.e. $\left.D(0)=\frac{1}{2} \cos ^{2}(\phi(0))\right) ; \phi(0)=60^{\circ} \mathrm{S} ; \lambda(0)=-10^{\circ}$; $V(0)=2 \alpha \lambda(0) / \sin (2 \phi(0))$ (solid curve) and $V(0)=0$ (dotted curve). The former curve is the special solution Eq. (2.14) while the latter (dotted) curve of Fig. 2 clearly demonstrates the oscillatory (i.e. stable) behaviour of the system near the special solution (2.14), i.e. when $V(0) \neq 2 \alpha \lambda(0) / \sin (2 \phi(0))$. Since $U(0)=0$ in both cases, the initial condition $V(0)=0$ in the oscillatory curve implies, according to Eq. (2.5), that $\mathrm{H}=\frac{1}{2} \alpha \lambda(0)^{2}$ is the potential energy. Thus, at short times the potential energy can 


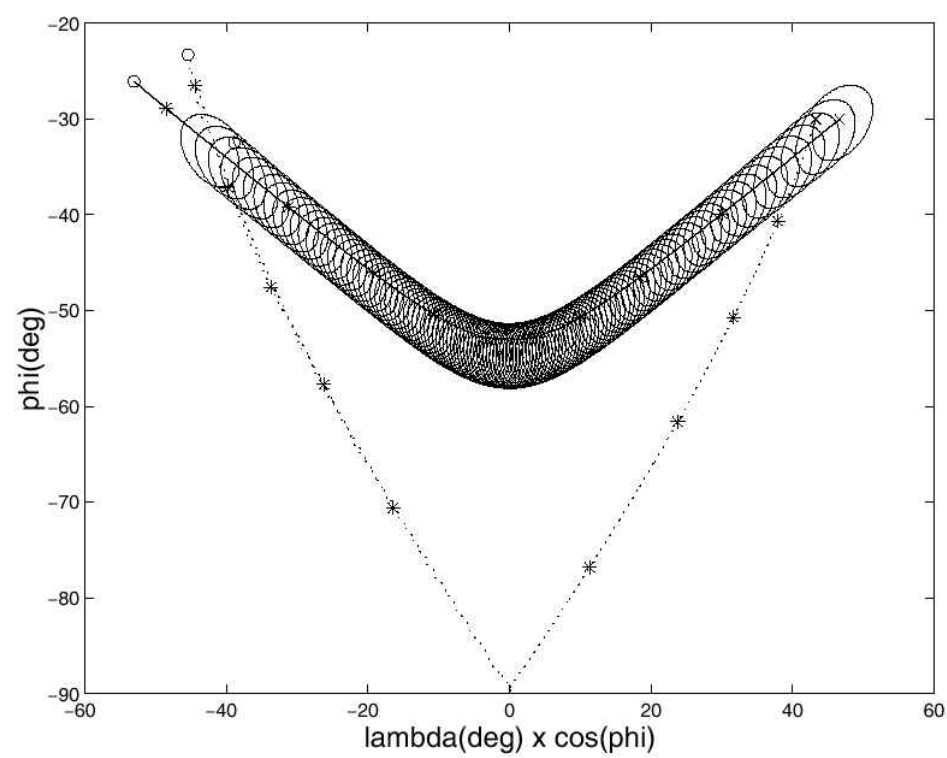

Figure 3. The midlatitudes averaged trajectory (solid curve inside spiral), the actual trajectory (solid, spiralling curve) and the geostrophic special solution (dotted curve) for $\alpha=0.001$. The averaged trajectory accurately filters out the (inertial) oscillations from the actual trajectory. The geostrophic solution provides a very poor estimate of the actual trajectory (and even reaches the South Pole) since $V(0)=-0.04$, which is twenty-times the initial northward velocity of the geostrophic special solution $V_{\mathrm{s}}=-0.002(=2 \alpha \lambda(0) / \sin (2 \phi(0))$. The other initial conditions are: longitude $\lambda(0)=50^{\circ} \mathrm{E}$, latitude $\phi(0)=30^{\circ} \mathrm{S}$ and zonal velocity component $U(0)=0$ (i.e. angular momentum $D(0)=0.125$ for the averaged trajectory). Final time is 920 time units (2.5 months), stars mark 100 time-unit (8-day) intervals, and initial and final locations are marked by ' $x$ ' and 'o'. See text for details and discussion.

only decrease, to enable the increase in the kinetic energy, i.e. the eddy must start its trajectory with positive $U$ so $\frac{1}{2} \alpha \lambda^{2}$ can decrease.

The kinetic energy that develops when the potential energy decreases is divided into two components: the meridional geostrophic motion of the special solution (2.14), and the oscillations near it. The only way to observe only the former component (the solid curve of Fig. 2) is to impose the geostrophic velocity as the initial velocity. This curve of Fig. 2 also demonstrates the analytical result regarding the second order westward/eastward drift at latitudes poleward/equatorward of $45^{\circ}$, which are masked by the oscillations in the thin-solid curve. In the same way that equatorward motion occurs only along the channel's western flank (Fig. 2), poleward motion can only occur along the channel's eastern flank where $\lambda>0$, so $D$ decreases $\left(D_{t}=-\alpha \lambda\right)$ to enable the increase in latitude.

As was noted above, the averaged trajectory of section 2(d) is also valid only in midlatitudes, and it provides a more accurate estimate than the special solution there when the initial velocity is not geostrophic. A numerical demonstration of the improvement afforded by the averaged trajectory over the special (geostrophic) solution is given in Fig. 3 for the case where $V(0) \neq V_{\mathrm{s}}=2 \alpha \lambda(0) / \sin (2 \phi(0))$. As discussed above, in this case the geostrophic solution (dotted curve) approximates the actual trajectory (spiralling solid curve) very poorly, while the averaged trajectory (solid curve inside spiral) reproduces the average of the actual trajectory over the inertial oscillations with impressive accuracy. 
(a)

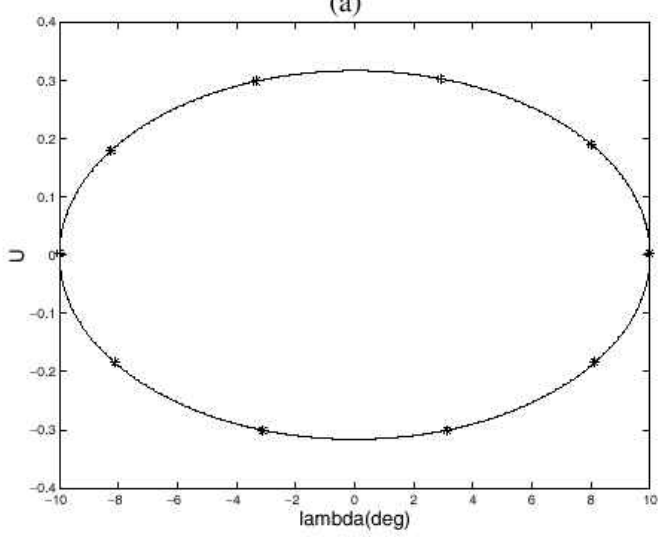

(b)

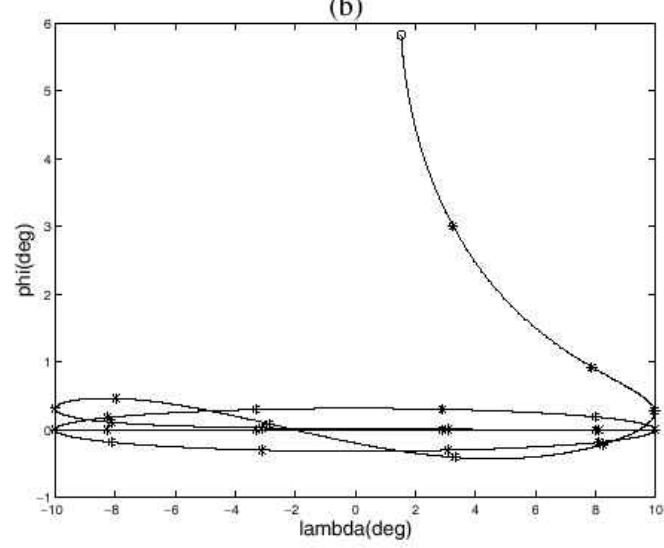

Figure 4. Equatorial oscillations. (a) Right at the equator: latitude $\phi(0)=0$ (shown in (longitude, $\lambda, U=D-\frac{1}{2}$ ) phase space, where $U$ is zonal velocity component and $D$ angular momentum, since the $(\lambda, \phi)$ curve is a straight line); the final time is 200 (16 days) and stars mark one-tenth of that time. (b) Near the equator: $\phi(0)=0.001^{\circ}$ (100 $\mathrm{m}$ north of the equator) final time is 350 (28 days) and asterisks marks 20 time unit (1.6-day) intervals. In both cases: $\lambda(0)=-10^{\circ}$; meridional velocity component $V(0)=0=U(0)$ and $\alpha=0.001$ (see text) were used. The instability of the equatorial regime is clearly evident in the growing meridional extent of the trajectory in (b), which occurs on the westward segment only. Initial and final locations are marked by ' $\mathrm{x}$ ' and 'o'.

\section{EQUATORIAL TRAJECTORIES}

As was shown above, the motion of the eddy along the equator is oscillatory with frequency $\alpha^{\frac{1}{2}}$. The motion along the eastward segment of the oscillation is stable, so the eddy encounters no problem traversing to the channel's eastern flank. In contrast, the motion along the oscillation's westward segment is unstable, so during this segment an expulsion of the eddy from the equatorial oscillation regime is expected to occur. These results are now confirmed numerically.

\section{(a) Numerical confirmation}

The numerical computation of the oscillations near the equator shown in Fig. 4 clearly confirms the analytic conclusions reached above. Right on the equator, the eddy oscillates with a $\alpha^{\frac{1}{2}}$ frequency, as is evident from the phase space plot in Fig. 4(a) (the corresponding $(\lambda, \phi)$ plot is a trivial straight line), where kinetic energy is continuously exchanged with potential energy (while, of course, the Hamiltonian's value remains constant at all times). When the trajectory originates slightly off the equator (e.g. $0.001^{\circ}$ latitude-a mere $100 \mathrm{~m}$ ), on the channel's western flank, the results shown in Fig. 4(b) demonstrate that the eddy successfully completes the eastward segment of the oscillation while remaining close to the equator. This is consistent with the analytic result regarding the stability of the special solution along its eastward segment. By comparison, in the return (westward) segment of the oscillation the trajectory is unstable $\left(D<\frac{1}{2}\right)$ and in agreement with this instability, the eddy in Fig. 4(b) is totally repelled from the equator during the westward segment of its second oscillation and never completes the oscillation. Similar behaviour is also encountered in the numerical simulations on the equatorial $\beta$-plane carried out by NB and Borisov and Nof (1998). 


\section{THE COMBINED TRAJECTORY-RELEVANCE TO OBSERVATIONS}

The presence of the AABW throughout the western South Atlantic is well documented. The motion of this dense water can easily be explained, based on a balance of forces between the eastward-directed pressure gradient force on the channels' western flank and the westward-directed Coriolis force associated with an equatorward flow in the southern hemisphere. An alternative, angular momentum, paradigm of the same motion follows from the equation $D_{t}=-\alpha \lambda$, which suggests that the only way for $D$ to increase $\left(D_{t}>0\right)$ is for the eddy to move along the western flank of the channel where $\lambda<0$. The continuous increase in $D$ at fixed $\lambda$, when $U^{2}$ is not free to grow beyond a maximal value set by the energy conservation, is accomplished by increasing $\cos (\phi)$, i.e. decreasing $\phi$.

When the AABW eddy is located sufficiently close to the equator along the western flank its angular momentum $D$ has reached a value close to $\frac{1}{2}$, and it is subject to the equatorial oscillation dynamics. Along the initial eastward segment of the oscillation, the speed, proportional to $\alpha^{\frac{1}{2}}$, attains much larger values than in the midlatitude motion, and $U$ and $D$ undergo a complete cycle from their respective initial values of 0 and $\frac{1}{2}$ to their maximal values at the channel's centre-line and back to 0 and $\frac{1}{2}$ values at the eastern longitude. Since the eastward segment is stable, the latitude $\phi(t)$ remains near zero. Upon commencing the westward segment of its oscillation the motion becomes unstable, since $D$ is decreased $(U<0)$ to values smaller than $\frac{1}{2}$. On the unstable westward segment, the eddy can either be expelled from the equator (to the midlatitudes of one of the two hemispheres) or complete its equatorial oscillation and return to the western flank while increasing the $(\phi, V)$ values relative to their values on the eastward segment. As was shown in Fig. 4, both scenarios occur in dissipation-free simulations of system (2.2) with equal probabilities. Since the expulsion of the eddy from the equator takes place only on the westward segment, it is clear why no AABW water can be found in the western North Atlantic-eddies can easily leave it but cannot return to it.

Up to this point, the dissipation (mixing) of the eddy's dense water is completely ignored, but it can be linked either to the shear associated with the eddy's motion in the quiescent overlying ocean water or to bottom friction. A large fraction of this mixing is presumably induced by the eddy's swirl velocity and not only by the translation velocity, but the former cannot easily be parametrized in the present model while the latter can. Thus, to get an appreciation of the effect of mixing in the context of system (2.2) dissipation terms negatively proportional to the eddy's velocity $(-\mu U,-\mu V)$ are added to the momentum $(2 \cdot 2 \mathrm{a}, \mathrm{b})$. Accordingly, the steady state in which the eddy does not move at all is considered as a state complete of mixing. This naive approximation can be expected to model properly the effect of dissipation, provided it is small enough so that the structure of the system differs only slightly from a frictionless model (2.2) i.e. $\mu \ll 1$. In this case the trajectory will slowly spiral to the steady state along the channel's centre-line. If this naive mixing scenario is valid, then it can be anticipated that dissipation occurs primarily when the eddy's translation velocity is largest, namely along the two equatorial oscillation segments.

As was noted in subsection 2(b), a frictionless time-dependent equatorial motion has a tendency to drift eastward. This inherent eastward drift tangles with the frictionless westward-directed equatorial trajectory, so the addition of dissipation makes the westward segment of the equatorial oscillation even more difficult to complete. This increases the likelihood of the eddy leaving the equator, thus commencing the midlatitude, geostrophic, poleward directed motion early during its westward segment. An example of the combined trajectory when dissipation (with $\mu=0.001$ ) is included in (2.2) is 
(a)

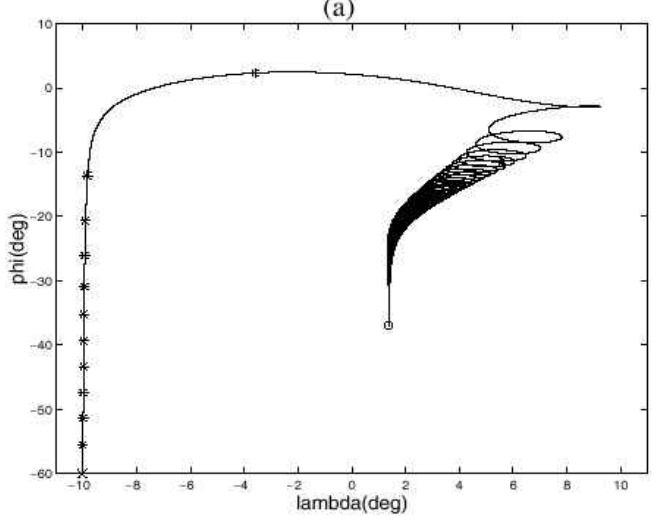

(b)

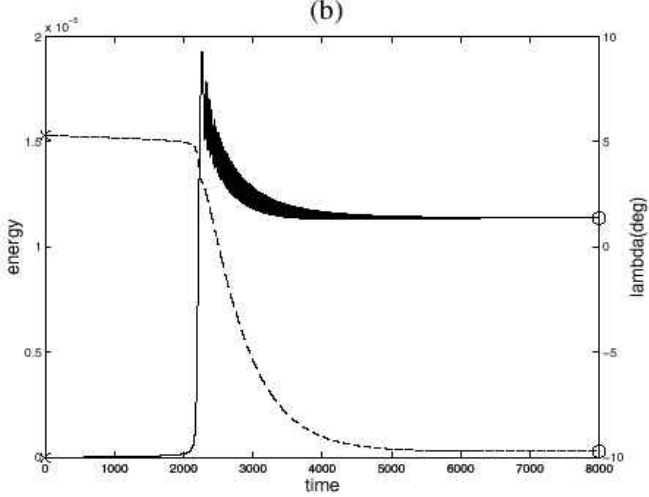

Figure 5. The combined, 8000 time-unit (21-month) long, trajectory that consists of Antarctica-to-equator, equatorial oscillation and equator-to-pole regimes. Small dissipation terms: $(-0.001 U,-0.001 V)$, where $U$ and $V$ are zonal and meridional velocity components, respectively, are added to the momentum equations $(2.2 \mathrm{a}, \mathrm{b})$ to account for mixing of the dense eddy water with the surrounding water: (a) latitude, longitude $(\lambda, \phi)$ trajectory; stars mark 200 time units (16 days) for the first 190 days; (b) energy (dashed curve, left abscissa) and longitude (solid curve, right abscissa) versus time; dissipation occurs mostly in the regime of the trajectory where the velocity is large i.e. in the equatorial oscillation regime (the regime with rapid longitudinal excursions). The energy scales on the square of the velocity scale, $2 \Omega R^{2}=0.865 \times 10^{-6} \mathrm{~m}^{2} \mathrm{~s}^{-2}$.

shown in Fig. 5. The tendency of the eddy to leave the equator early during the westward segment of the oscillation, i.e. while it is located on the eastern flank, is clear in the geographic trajectory, Fig. 5(a). Dissipation of energy is dramatic both during (i.e. the eastward segment of) the equatorial oscillation and near the midlatitude motion, whereas in the steady midlatitude geostrophic segments energy loss is negligible due to the low velocities involved. This correlation between changes in energy and longitude is clearly evident in Fig. 5(b).

Although the analytical results derived in this study were obtained by treating deep eddies as particles, it cannot be applied straightforwardly to the trajectories of deep SOFAR* floats (that are definitely particles) reported by Richardson and Schmitz (1993) and by Richardson and Frantantoni (1999). The reason is that the particles in the present model are denser than the surrounding water so they move right at the ocean floor, while SOFAR floats are ballasted to move along fixed isobaric surfaces above the bottom, and the sloping bottom does not affect the dynamics of overlying floats as it does for bottom trapped eddies.

The sensitive transition from midlatitude geostrophic flow to the equatorial oscillation regime is the cause of the 'splitter' effect encountered in the numerical simulation of NB on the equatorial $\beta$-plane. In these simulations, both a cloud of particles and a geostrophic current moving along the channel's bottom were split into two parts (thus the term 'splitter') upon reaching the equator, each continuing its motion in another hemisphere. Equatorial dynamics is, in fact, an absolute barrier to cross-equatorial motion along the channel's western flank, while along its eastern flank the same dynamics allows some eddies to escape meridionally to either of the two hemispheres while some

\footnotetext{
* SOFAR floats are neutrally buoyant floats that transmit acoustic signals captured by hydrophones placed at the depth of the SOFAR (SOund Fixing And Ranging) channel. The low frequency $(\ll 1000 \mathrm{~Hz})$ signals can travel great distances $(>1000 \mathrm{~km})$ in the sound channel and, by measuring the travel times of a signal to an array of hydrophones located at the channel's depth along the ocean's shores, one can calculate the float's exact location. The float's trajectory is then constructed from the time series of its location.
} 
(a)

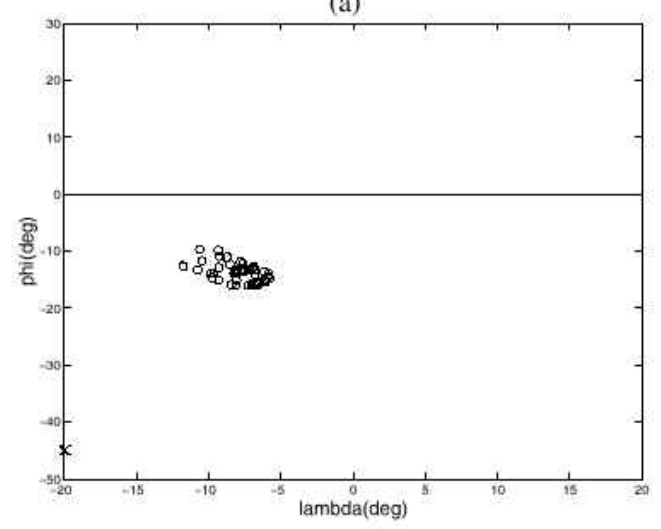

(b)

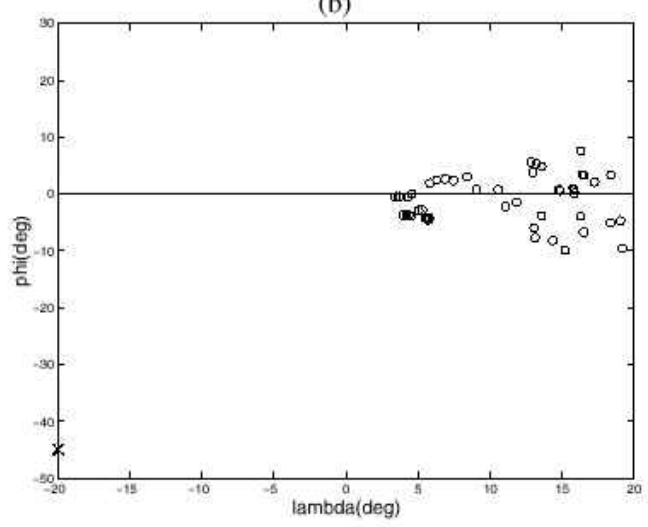

(c)

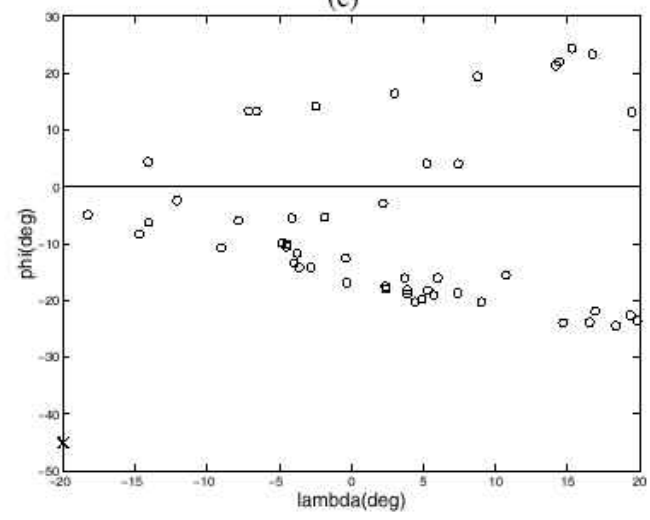

Figure 6. Final locations (open circles) of 50 eddies launched in a $5 \mathrm{~km}^{2}$ square centred on latitude $\lambda=45^{\circ} \mathrm{S}$, longitude $\phi=20^{\circ} \mathrm{W}$ (marked x) after: (a) 2600 time units (7 months); (b) 3000 time units ( 8 months); and (c) 3500 time units ( 9 months). All eddies are initialized with their geostrophic velocity and the value of $\alpha$ (see text) is $5 \times 10^{-4}$. Note that speed increases on the equator, and that both fast dispersal and migration to the northern hemisphere occur on the equatorial oscillation's westward segment.

eddies can return westward so the cloud of particles splits. This splitting effect of the equator on its eastern flank results (at least for particles) from the non-integrability (i.e. chaotic nature) of system (2.6). A demonstration of the splitting effect, as well as the sensitivity of system (2.6) to the precise initial conditions, is given in Fig. 6 where the position of a cloud of 50 particles is calculated at different times. The 50 particles were launched in a $5 \times 5 \mathrm{~km}^{2}$ square centred at $45^{\circ} \mathrm{S}, 20^{\circ} \mathrm{W}$, each particle with its own geostrophic velocity. In the first 7 months (2600 time units) the distance between particles grew to $\mathrm{O}(500) \mathrm{km}$ only (Fig. 6(a)), and in that period the entire cloud reached the equator along the eastern flank, and completed one equator oscillation with little dispersal. In the next months $(t=3000)$ the cloud crossed the channel from west to east along the equator with a moderate dispersal, particles are spread over $1500 \mathrm{~km}$ (Fig. 6(b)), but upon commencing its westward segment 1 month later (at $t=3500$ or 9 months after launch) the particles are O(6000) $\mathrm{km}$ apart (Fig. 6(c))—with some particles still located along the equator while others are at higher latitudes in both hemispheres. As expected, the dispersal occurs mostly on the westward segment of the equatorial oscillation, and the particle speeds along this segment are much higher than their speed along the Antarctica-to-equator part. The sensitivity of the final location 
(and the hemisphere where the particle finds itself) to the precise value of the initial location is a manifestation of the existence of chaotic bands that typifies non-integrable Hamiltonian systems such as (2.6). The same is true for the intermittent, rather than smooth/continuous, dispersal.

\section{ACKNOWLEDGEMENTS}

The US-Israel Bi-National Science Foundation provided financial support for this work via a research grant to the Hebrew University of Jerusalem and Florida State University. The comments of two anonymous reviewers greatly improved the presentation of this work.

Borisov, S. and Nof, D.

Dvorkin, Y. and Paldor, N.

Nof, D. and Borisov, S.

Paldor, N.

Paldor, N. and Killworth, P. D.

Paldor, N. and Sigalov, A.

Richardson, L. P. and Schmitz, W. J. 1993

Richardson, L. P. and Frantantoni, D. M.

Stommel, $\mathrm{H}$.

Stommel, H. and Arons, A. B.

Whitehead, J. A. and Worthington, L. V.

\section{REFERENCES}

1998 Deep, cross-equatorial eddies. Geophys. Astrophys. Fluid Dyn., 87, 273-310

1999 Analytical considerations of Lagrangian cross-equatorial flow. J. Atmos. Sci., 56, 1229-1237

1998 Inter-hemispheric oceanic exchange. Q. J. R. Meteorol. Soc., 124, 2829-2866

2001 The zonal drift associated with time-dependent particle motion on the earth. Q. J. R. Meteorol. Soc., 127, 2435-2450

1988 Inertial trajectories on the rotating earth. J. Atmos. Sci., 45, 40134019

2001 The mechanics of inertial motion on the earth and on a rotating sphere. Physica D, 1601, 29-53

Deep cross-equatorial flow in the Atlantic measured with SOFAR floats. J. Geophys. Res., 98(C5), 8371-8387

1999 Float trajectories in the deep western boundary current and deep equatorial jets of the tropical Atlantic. Deep-Sea Res. II, 46, 304-333

1958 The abyssal circulation. Deep-Sea Res., 5, 80-82

1960a On the abyssal circulation of the world ocean: I. Stationary planetary flow patterns on a sphere. Deep-Sea Res., 6, 140-154

$1960 \mathrm{~b}$ On the abyssal circulation of the world ocean: II. An idealized model of the circulation pattern and amplitude in oceanic basins. Deep-Sea Res., 6, 217-233

1982 The flux and mixing rates of Antarctic Bottom Water within the North Atlantic. J. Geophys. Res., 87(C10), 7903-7924 